\title{
Erratum to: Synergism between Enantiomers Creates Species-Specific Pheromone Blends and Minimizes Cross-Attraction for Two Species of Cerambycid Beetles
}

\author{
Linnea R. Meier ${ }^{1}$ - Yunfan Zou ${ }^{2}$ - Jocelyn G. Millar ${ }^{2}$. Judith A. Mongold-Diers ${ }^{1}$ • \\ Lawrence M. Hanks ${ }^{1}$
}

Published online: 26 November 2016

(C) Springer Science+Business Media New York 2016

Erratum to: J Chem Ecol

DOI 10.1007/s10886-016-0782-z

In the original version of this article, a line on the fourth page, third paragraph, has the retention times for $(R)$ - and $(S)$ fuscumol acetate reversed. The correct sentence should read, "Structures were confirmed by coinjection of an aliquot of aeration extract with the mixture of synthetic stereoisomers (Millar et al. 2009); (S) - and (R)-fuscumol acetate (retention times 51.33 and $51.72 \mathrm{~min}$, respectively) and $(Z)$ - and $(E)$ geranylacetone were resolved to baseline (retention times 14.53 and $14.74 \mathrm{~min}$, respectively)." The authors regret this error.

The online version of the original article can be found at $\mathrm{http}: / / \mathrm{dx}$.doi. org/10.1007/s10886-016-0782-z

Lawrence M. Hanks

hanks@life.illinois.edu

1 Department of Entomology, University of Illinois at

Urbana-Champaign, 320 Morrill Hall, 505 South Goodwin Avenue,

Urbana, IL 61801, USA

2 Department of Entomology, University of California, Riverside, CA 92521, USA 